\title{
Establish a Scoring Model for High-Risk Population of Gastric Cancer and Study on the Pattern of Opportunistic Screening
}

\author{
Wei Tao $\left(\mathbb{D},{ }^{1}\right.$ Hai-Xia Wang $\left(\mathbb{D},{ }^{2}\right.$ Yu-Feng Guo, ${ }^{1}$ Li Yang, ${ }^{1}$ and Peng $\mathrm{Li}^{1}$ \\ ${ }^{1}$ Department of Gastroenterology, General Hospital of Ningxia Medical University, Yinchuan 750004, China \\ ${ }^{2}$ Ningxia Medical University, Yinchuan 750004, China \\ Correspondence should be addressed to Wei Tao; nyfytaowei@163.com and Hai-Xia Wang; 957376775@qq.com
}

Received 7 May 2020; Revised 8 September 2020; Accepted 16 September 2020; Published 30 September 2020

Academic Editor: Kazuhiko Uchiyama Copyright $\odot 2020$ Wei Tao et al. This is an open access article distributed under the Creative Commons Attribution License, which
permits unrestricted use, distribution, and reproduction in any medium, provided the original work is properly cited.

\begin{abstract}
Objective. To investigate and study the related risk factors of gastric cancer (GC) patients, to establish a high-risk scoring model of GC by multiple logistic regression analysis, and to explore the establishment of a GC screening mode with clinical opportunistic screening as the main method, and by using the pattern of opportunistic screening to establish the screening of high-risk GC patients and the choice of screening methods in the clinical outpatient work. Methods. Collected the epidemiological questionnaire of 99 GC cases and 284 non-GC patients (other chronic gastric diseases and normal) diagnosed by the General Hospital of Ningxia Medical University from October 2017 to March 2019. Serum pepsinogen (PG) levels were measured by enzyme-linked immunosorbent assay (ELISA) and confirmed Helicobacter pylori ( $\mathrm{Hp}$ ) infection in gastric mucosa tissues by Giemsa staining. Determined the high-risk factors and established a scoring model through unconditional logistic regression model analysis, and the ROC curve determined the cut-off value. Then, we followed up 26 patients of nongastric cancer patients constituted a validation group, which validated the model. Results. The high-risk factors of GC included age $\geq 55$, male, drinking cellar or well water, family history of GC, $\mathrm{Hp}$ infection, $\mathrm{PGI} \leq 43.6 \mu \mathrm{g} / \mathrm{L}$, and PGI/PGII $\leq 2.1$. Established the high-risk model: Y $=\mathrm{A} \times$ age $+30 \times$ gender $+30 \times$ drinking water $+30 \times$ Hp infection $+50 \times$ family history of $\mathrm{GC}+\mathrm{B} \times \mathrm{PG}$ level. The ROC curve determined that the cut-off value for high-risk GC population was $\geq 155$, and the area under the curve (AUC) was 0.875 , the sensitivity and specificity were $87.9 \%$ and $71.5 \%$. Conclusions. According to the risk factors of GC, using statistical methods can establish a high-risk scoring model of GC, and the score $\geq 155$ is divided into the screening cut-off value for high-risk GC population. Using this model for clinical outpatient GC screening is cost-effective and has high sensitivity and specificity.
\end{abstract}

\section{Introduction}

Gastric cancer (GC) remains one of the most common neoplasms in the world [1]. China is a country with a high incidence of GC, with an annual incidence rate of about $19.62 / 100,000$, and a mortality rate of about $13.44 / 100,000$ [2]. GC screening is still considered to be the most direct and effective intervention [3]. However, China's large population and lack of medical resources cannot implement largescale gastroscopy screening. Finding and establishing screening methods and standards for screening high-risk populations of GC in line with China's national conditions have important practical significance. Studies have shown $[4,5]$ that the carcinogenesis and development of GC were caused by a combina- tion of external environmental factors such as population, lifestyle, diet, infection, social economy, and internal genetic factors such as a family history of tumors. In this article, we have established a scoring model for high-risk populations of GC through statistical logistic regression analysis and receiver operating characteristic (ROC) curve through the risk factors of GC, having combined the patients' PG levels and Hp infection rates, and to explore the opportunistic screening methods for GC suitable for China's national conditions.

\section{Methods and Materials}

2.1. General Information. By case-control study, we collected 383 patients with the gastric disease diagnosed by the 
outpatient department of Gastroenterology, Affiliated Hospital of Ningxia Medical University from October 2017 to March 2019, and signed the informed consents, while collecting $5 \mathrm{ml}$ of fasting venous blood. All patients were diagnosed by gastroscopy and histopathology, including 99 cases of GC, 284 cases of non-GC ( 88 cases of chronic superficial gastritis, 104 cases of chronic atrophic gastritis, and 92 cases of gastric ulcer). The diagnosis of GC and chronic gastric disease was based on the diagnostic criteria for gastric mucosal lesions of the "Newly-edited Standards for the Diagnosis and Treatment of Common Malignant Tumors (Gastric Cancer Volume)" by the Chinese Anti-Cancer Association. After that, we followed up 48 of nongastric cancer patients randomly, 22 of whom did not have an electronic gastroscopy examination (EGE), so they were excluded. The remaining 26 patients performed an EGE and pathological tissue biopsy again to form a validation group, and the established model was applied to the validation group.

2.2. Epidemiological Questionnaire. We conducted face-toface questionnaires for each research-studied subjects. The content included gender; age; ethnic group; eating habits such as eating pickled products, fresh vegetables, and drinking water; current medical history; past medical history; and family history of gastrointestinal cancer. Among them, according to the total amount of fresh vegetables eaten daily, it was divided into a low amount group $(<0.25 \mathrm{~kg} /$ day $)$, a medium amount group (0.25-0.5 kg/day), and a high amount group ( $>0.5 \mathrm{~kg} /$ day); the situation of edible pickled products was divided into occasional ( $<3$ times/week) and often $(>3$ times/week); the situation of drinking water was divided into tap water, well water, or cellar water.

2.3. ELISA. The Hp infection status, PGI level, and the ratio of PG I to II (PGR) of all studied subjects were measured. The double-antibody sandwich ELISA kit of Rigor Bioscience Development TLD was used to determine the content of fasting serum pepsinogen subgroups PG I and PG II in these subjects.

2.4. Giemsa Staining. Histological diagnosis of $\mathrm{Hp}$ infection was performed with Giemsa staining kits from Bioss Antibodies under a microscope and combined with rapid detection of urokinase. Both tests were positive, so the subjects were positive for $\mathrm{Hp}$ infection.

2.5. Statistical Analyses. Univariate analysis performed on various factors in the epidemiological questionnaire and multiple logistic regression analysis was used to determine the statistically meaningful risk factors, and the regression coefficient $\beta$ of each independent variable was obtained. Then calculated the multiple of the $\beta$ value of other independent variables with the smallest $\beta$ value as the base, which was the corresponding weight score of each independent variable, and established a high-risk scoring model on this basis. The case group and the control group were scored according to the above scoring model, and the cut-off value with higher predictive value was determined by the ROC curve analysis. All data were processed and analyzed by SPSS 11.5 software. $P<0.05$ was considered statistically significant.
TABLE 1: Mono factor analysis results of influencing factors.

\begin{tabular}{lcc}
\hline Factors & $\chi^{2}$ & $P$ value \\
\hline Gender (X1) & 10.620 & 0.001 \\
Age (X2) & 46.958 & 0.001 \\
Ethnic group (X3) & 0.038 & 0.845 \\
Drinking water (X4) & 24.913 & 0.001 \\
Fresh vegetables (X5) & 4.142 & 0.126 \\
Pickled products (X6) & 6.422 & 0.011 \\
Hp infection (X7) & 27.800 & $<0.0005$ \\
Family history (X8) & 22.466 & 0.001 \\
PGR (X9) & 38.287 & $<0.0005$ \\
\hline
\end{tabular}

\section{Results}

3.1. Mono Factor Analysis Results. Through the analysis of the single-factor chi-square test, in the surveyed factors, the gender was male, the age was $\geq 55$ years, the ethnic group was Hui, the drinking water was well water or cellar water, often ate pickled products, $\mathrm{Hp}$ infection, and a family history of gastrointestinal cancer, $\mathrm{PGI} \leq 43.6 \mu \mathrm{g} / \mathrm{L}$, and $\mathrm{PGR} \leq 2.1$ were the influencing factors of GC carcinogenesis $(P<0.05)$ (Table 1).

3.2. Multifactor Logistic Regression Model Coding. For the convenience of analysis, all variables were set as categorical variables; for some continuous variables such as age and PG, according to the research data, we set corresponding cut-off values, then which were converted into categorical variables, and multiple logistic regression analysis was performed, such as PGI $\leq 43.6 \mu \mathrm{g} / \mathrm{L}$ and $\mathrm{PGR} \leq 2.1$. The specific codes were shown in Table 2.

3.3. Multivariate Analysis Results. Table 3 showed that age, gender, drinking water, Hp infection, PGR, and family history were the high-risk factors affecting GC through multivariate conditional logistic regression analysis, among PGR was the most main influencing factor.

3.4. Establish a High-Risk Model of GC. To score patients in clinical work more effectively, the two continuous variables of age and PG level were treated with dummy variables, and then multivariate conditional logistic regression analysis (Table 4) was performed to obtain the regression coefficients $\beta$ of factors influencing the incidence of GC, using the smallest $\beta$ value (0.208) as the base, calculated the multiples of the $\beta$ value of the other independent variables compared to it, then multiplied it by 10 , which was the corresponding weight score of each independent variable, and used this as a basis for each risk factor assigned values (Table 5) to establish a GC high-risk scoring model, and finally this model as follows:

$\mathrm{Y}=\mathrm{A} \times$ age $+30 \times$ gender $+30 \times$ drinking water $+30 \times$ $\mathrm{Hp}$ infection $+50 \times$ family history of $\mathrm{GC}+\mathrm{B} \times \mathrm{PG}$ level (when $35<$ age $\leq 45, A=20 ; 45<$ age $\leq 55, A=40$; when 55 < age $\leq 65, A=70$; when age $>65, A=80$; when $\mathrm{PGI} \leq 43.6$ $\mu \mathrm{g} / \mathrm{L}$ and $\mathrm{PGR}>2.1, B=10 ; \mathrm{PGI}>43.6 \mu \mathrm{g} / \mathrm{L}$ and when 
TABLE 2: Logistic regression model coding.

\begin{tabular}{lccc}
\hline Variables & Influencing factors & Quantitative method \\
\hline X1 & Gender & $0:$ female & $1:$ male \\
X2 & Age & $0:<55$ & $1: \geq 55$ \\
X3 & Ethnic group & $0:$ Han nationality & $1:$ Hui nationality \\
X4 & Drinking water & $0:$ tap water & $1:$ well water or cellar water \\
X5 & Fresh vegetables (kg/day) & $1:<0.25$ & $3:>0.5$ \\
X6 & Pickled products & $0:$ occasional & $2: 0.25-0.5$ \\
X7 & Hp infection & $0:$ negative & $1:$ often \\
X8 & Family history & $0:$ no & $1:$ positive \\
X9 & PGR & $0:$ no & $1:$ yes \\
\end{tabular}

TABLE 3: Results of logistic regression analysis.

\begin{tabular}{|c|c|c|c|c|c|c|c|}
\hline \multirow{2}{*}{ Factors } & \multirow{2}{*}{$\mathrm{B}$} & \multirow{2}{*}{ SE } & \multirow{2}{*}{ Wald } & \multirow{2}{*}{ SIG } & \multirow{2}{*}{$\operatorname{Exp}(B)$} & \multicolumn{2}{|c|}{ OR $95 \%$ CI } \\
\hline & & & & & & Lower limit & Upper limit \\
\hline Gender & 0.811 & 0.327 & 6.137 & 0.013 & 2.250 & 1.185 & 4.275 \\
\hline Drinking water & 0.886 & 0.297 & 8.922 & 0.003 & 2.425 & 1.356 & 4.338 \\
\hline Hp infection & 0.781 & 0.372 & 4.417 & 0.036 & 2.184 & 1.054 & 4.523 \\
\hline Family history & 1.173 & 0.336 & 12.185 & 0.000 & 3.231 & 1.672 & 6.242 \\
\hline PG level & 1.752 & 0.229 & 34.240 & 0.000 & 5.768 & 3.207 & 10.374 \\
\hline Age & 1.462 & 0.305 & 23.029 & 0.000 & 4.314 & 2.375 & 7.838 \\
\hline
\end{tabular}

TABLE 4: Results of logistic regression analysis.

\begin{tabular}{|c|c|c|c|c|c|c|c|}
\hline \multirow{2}{*}{ Factors } & \multirow{2}{*}{$\mathrm{B}$} & \multirow{2}{*}{ SE } & \multirow{2}{*}{ Wald } & \multirow{2}{*}{ SIG } & \multirow{2}{*}{$\operatorname{Exp}(B)$} & \multicolumn{2}{|c|}{ OR 95\% CI } \\
\hline & & & & & & Lower limit & Upper limit \\
\hline Gender & 0.846 & 0.335 & 6.387 & 0.011 & 2.331 & 1.209 & 4.494 \\
\hline Drinking water & 0.929 & 0.305 & 9.295 & 0.020 & 2.532 & 1.384 & 4.602 \\
\hline Hp infection & 0.741 & 0.377 & 3.868 & 0.049 & 2.097 & 1.003 & 4.386 \\
\hline Family history & 1.281 & 0.348 & 13.577 & 0.000 & 3.602 & 1.822 & 7.121 \\
\hline Age & & & 23.533 & 0.000 & & & \\
\hline Age (1) & 0.408 & 0.835 & 0.238 & 0.625 & 1.503 & 0.293 & 7.719 \\
\hline Age (2) & 1.001 & 0.761 & 1.733 & 0.188 & 2.722 & 0.613 & 12.091 \\
\hline Age (3) & 1.932 & 0.733 & 6.955 & 0.008 & 6.904 & 1.642 & 29.606 \\
\hline Age (4) & 2.390 & 0.772 & 9.577 & 0.002 & 10.916 & 2.402 & 49.606 \\
\hline PG & & & 33.768 & 0.000 & & & \\
\hline PG (1) & 0.208 & 0.608 & 0.118 & 0.732 & 1.232 & 0.374 & 4.056 \\
\hline PG (2) & 0.706 & 0.521 & 1.836 & 0.175 & 2.025 & 0.730 & 5.619 \\
\hline PG (3) & 2.073 & 0.448 & 21.452 & 0.000 & 7.946 & 3.306 & 19.103 \\
\hline
\end{tabular}

PGR $\leq 2.1, B=30$; and when $\mathrm{PGI} \leq 43.6 \mu \mathrm{g} / \mathrm{L}$ and $\mathrm{PGR} \leq 2.1$, $B=80)$.

\subsection{Drawing of ROC Curves}

3.5.1. Scoring Patients with a High-Risk Scoring Model of GC. The two groups of patients were scored according to the above scoring criteria. The results (Table 6) showed that the control group had $121.30 \pm 57.363$ points and GC 208.89 \pm 47.313 points. The comparison between them was statistically significant $(P<0.001$, Mann Whitney test).
3.5.2. Draw the Modeling ROC Curve. To determine the cutoff value for the high-risk prediction of GC, the ROC curve was drawn according to the two groups of scores (Figure 1). According to the ROC curve, we preliminarily determined the score of the high-risk GC population as $\geq 155$, the AUC was 0.875 , the sensitivity and specificity were $87.9 \%$ and $71.5 \%$, and the Youden index was 0.594.

3.5.3. Analysis of the Validation Group. In the validation group, there were 6 cases of the nonhigh-risk group and 20 cases of the high-risk group. The results showed that no 
TABLE 5: High-risk scoring model for identification of GC and nontumor diseases of the digestive system.

\begin{tabular}{lcc}
\hline Factors & Score \\
\hline Age & Age $\leq 45$ & 20 \\
Age (1) & $45<$ age $\leq 55$ & 40 \\
Age (2) & $55<$ age $\leq 65$ & 70 \\
Age (3) & Age $>65$ & 80 \\
Age (4) & & 30 \\
Gender & & 30 \\
Drinking water & & 30 \\
Hp infection & & 50 \\
Family history & & \\
PG level & PGI $\leq 43.6 \mu \mathrm{g} / \mathrm{L}$ and PGR $>2.1$ & 10 \\
PG (1) & PGI $>43.6 \mu \mathrm{g} / \mathrm{L}$ and PGR $>2.1$ & 30 \\
PF (2) & PGI $\leq 43.6 \mu \mathrm{g} / \mathrm{L}$ and PGR $>2.1$ & 80 \\
PG (3) & &
\end{tabular}

TABLE 6: Scores of GC group and non-GC group.

\begin{tabular}{lcc}
\hline Groups & $N$ & Score \\
\hline GC group & 99 & $208.89 \pm 47.313$ \\
Non-GC group & 284 & $121.30 \pm 57.363^{1}$ \\
\hline
\end{tabular}

${ }^{1} P<0.001$ (compared with GC group).

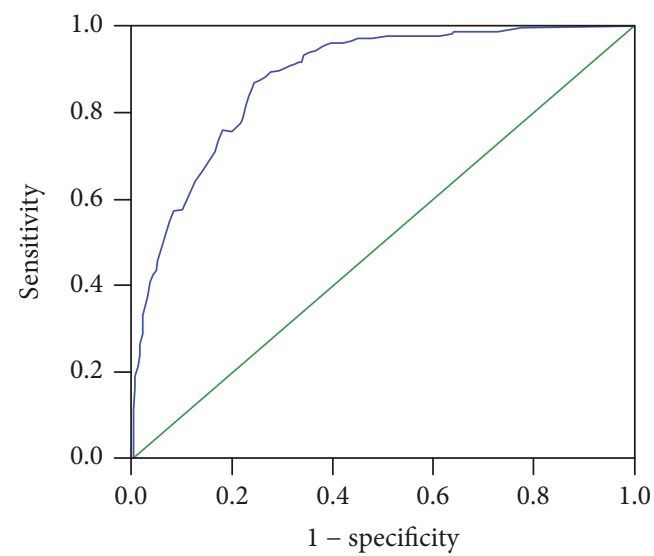

FIGURE 1: ROC curve of risk factor score.

malignant lesions were found in the nonhigh-risk group. There were 4 patients with GC in the high-risk group, including 1 case of stomach angle cancer, 2 cases of cardia cancer, and 1 case of gastric antrum cancer (Table 7). The pathological types were well-differentiated adenocarcinoma, moderate-well-differentiated adenocarcinoma, and poorly differentiated adenocarcinoma. After surgery, pathological examination confirmed that all tumor stages were T1N0M0, so the diagnosis rate of our model for early gastric cancer is $15.4 \%(4 / 26)$. The newly established model was applied to the validation group, and the ROC curve (Figure 2) showed that AUC was $0.883(P<0.001,95 \%$
TABLE 7: Compare the number of GC patients in high-risk group and nonhigh-risk group.

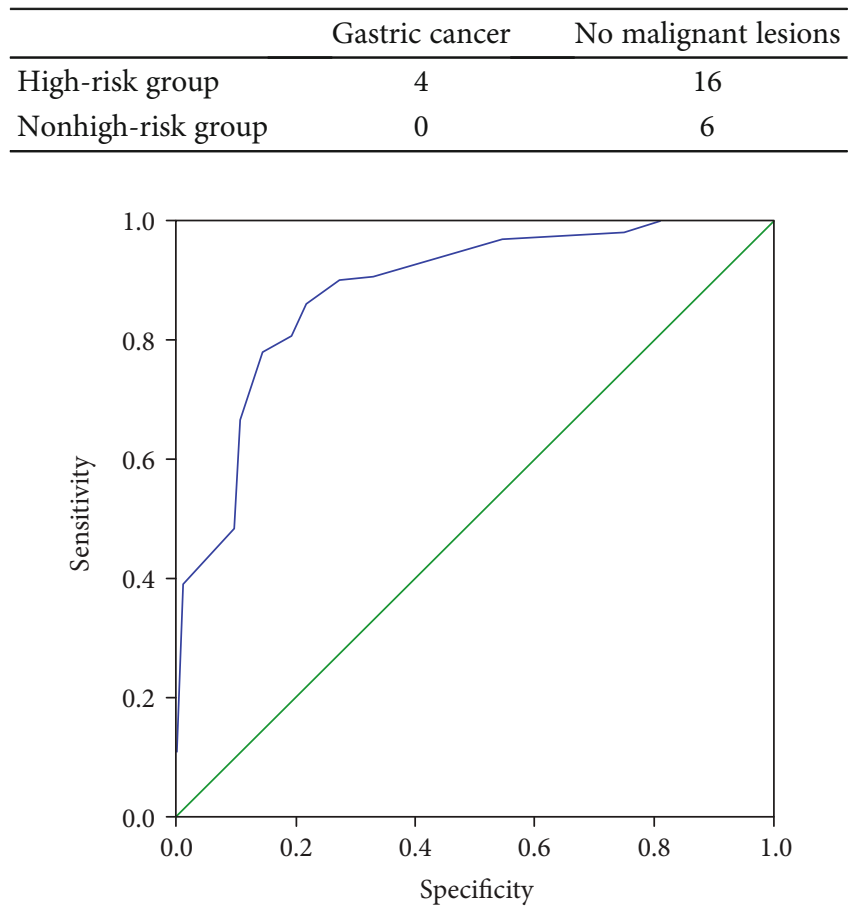

FIgURE 2: ROC curve of the validation group.

CI: 0.847-0.918), the Youden index was 0.644, the sensitivity was $86.2 \%$, and the specificity was $78.2 \%$.

3.6. Evaluation of the Model. The Goodness of fit test of the model was obtained by the Hosmer-Lelneshow (HL) test. The HL index of the model was 13.490 and $P=0.096$, indicating that the model fitted the data well. And the AUC of the validation group was 0.883 , the Youden index was 0.644 , the sensitivity was $86.2 \%$, and the specificity was $78.2 \%$, suggesting that the established high-risk scoring model for gastric cancer has good predictive value.

\section{Discussions}

Worldwide, the incidence of GC has been steadily declining in these years; nevertheless, GC is still a common malignant tumor [6], and its incidence and mortality rates are also one of the most common malignant tumors in China [2]. Ningxia is a higher incidence area of GC, and its incidence and mortality of GC are both at the forefront in the local malignant diseases [7]. The overall 5-year survival rate of GC is less than $50 \%$, and the cure rate of early GC can exceed $90 \%$, while the average 5 -year survival rate of advanced GC is less than about $30 \%$ [8]. Therefore, the purpose of GC screening is early detection, early diagnosis, and early treatment, which is of great significance for reducing the mortality rate [9]. However, China has a large population, an underdeveloped economy, and medical conditions, so it is difficult to carry out large-scale censuses. Opportunistic screening is also called individual screening or case finding. It is a kind of clinical screening, as well as a face-to-face examination, and it 
can be that the examinee takes the initiative to screen, or the doctor decides to screen according to the examinee's risk level. Because it is a clinical-based screening method that can be carried out all year round, its cost is lower, little staff is required, and the patient's compliance is far better than a national population-based GC screening, it is easier to implement. The carcinogenesis and development of GC are due to the comprehensive effect of multifactors, multistages, and multisteps, and some researches $[6,10,11]$ have shown that environmental carcinogens and genetic susceptibility are closely related factors for it. Studies by Kneller et al. [12] pointed out that regional differences, edible salted products, green vegetables, $\mathrm{Hp}$ infection, plasma selenium, plasma albumin levels, etc. were risk factors for GC. DenovaGutiérrez et al. [13] found that higher education levels, eradication of $\mathrm{Hp}$, more consumption of fresh fruits, vegetables, meat, etc. were positively correlated with GC, while alcohol, refined grains, sweets, soft drinks, etc. were significantly negatively correlated with GC. The further study of Thrift and El-Serag [14] have shown that $\mathrm{Hp}$ is the main risk factor for GC, and the amount of N-nitroso compounds (NOC) was related to GC, while the use of NSAIDs and statins, nonstarchy vegetables and fruits could lead to a further decrease in GC incidence and mortality. Previous studies $[15,16]$ have shown that the related risk factors of gastric cancer patients in our area were ethnic group, health and safety of drinking water, smoking, drinking, Hp infection, family history of GC, history of chronic digestive diseases, dietary factors (including fried food, high salt diet, pickled food, fresh vegetables, and fruits), eating habits (such as whether eating is too fast, whether three meals are regular or not), and other situations.

Our study combined previous studies on the risk factors of GC in Ningxia $[15,16]$ and reports of related domestic studies [4-6, 10-14, 17]. From the demographic factors, environmental factors, lifestyle, genetic susceptibility, and other factors combined with the clinical test results of $\mathrm{Hp}$ and PG to analyze the related factors of gastric carcinogenesis, then confirmed that gender, age, ethnic group, drinking water, pickled products, Hp infection, family history, and PGR were important risk factors for gastric carcinogenesis in our area. And starting from the risk factors of GC, statistical methods were used to establish a high-risk scoring model of GC, and then to explore the establishment of GC opportunistic screening methods suitable for China's national conditions. The results of our study showed that the gastric and non-GC groups had more significant differences in terms of gender, age, drinking water, $\mathrm{Hp}$ infection, family history of gastrointestinal cancer, and PGR, and among them, PGR is the most main factor. This conclusion was the same as other research results at home and abroad $[18,19]$. Based on this, according to the regression coefficients obtained by unconditional logistic regression analysis, we could calculate the weight score of each independent variable, finally establishing a high-risk scoring model. This is different from the cancer risk index scoring method established by Harvard University [20]. It mainly determines the score according to the OR value of each risk factor, and the purpose is to predict cancer carcinogenesis. However, the model we established was based on the weight of each factor in the unconditional logistic regression results to determine the score, showing the relative contribution of each risk factor to GC, which was helpful for diagnosis. Using this method to predict cancer carcinogenesis thorough risk assessment has been demonstrated [21-23], such as pancreatic cancer, breast cancer, and colorectal cancer, but there were few related studies on GC. To further evaluate the established high-risk scoring model, we drew the ROC working curve, and the results showed that a score of $\geq 155$ was an ideal cut-off value for distinguishing GC from non-GC. The AUC was 0.887; the sensitivity and specificity were $83.8 \%$ and $78.9 \%$. And the AUC of the validation group was 0.883 suggested that the established high-risk scoring model for gastric cancer has good predictive value. In the validation group, the diagnosis rate of our model for early gastric cancer reached $15.4 \%$. However, according to previous research reported that the diagnosis rate of early gastric cancer patients in China was $<10 \%$ [24], indicating that the scoring model we have established has a good value for early gastric cancer screening.

The establishment of the high-risk scoring model was based on the results of case-control studies, and all studied subjects were from clinical outpatients, including patients with common gastritis, peptic ulcers, and dyspepsia. This model fully considered the clinical practicality and provided new ideas for opportunistic screening of GC. Outpatient physicians can use the high-risk scoring model for GC to score patients in outpatient clinics, and then perform gastroscopy on high-risk groups with a score $\geq 155$, which is more likely to screen out GC patients. Close follow-up and observation of high-risk groups with negative gastroscopy and a score $\geq$ 155 are expected to increase the screening rate for early GC.

This model is simple, convenient, and economical, has good patient compliance, is easy to implement clinically, is easy to concentrate medical resources, and is expected to identify high-risk groups at an early stage, then to increase the detection rate of GC. However, in this study, due to the amount of sample selection is insufficient, whether the selected factors of GC are comprehensive and whether these factors have collinearity and the problem of confounding factors, so the conclusion should be further explored. At the same time, because this study was conducted based on a case-control study, the proportion of patients with advanced GC was relatively higher. Therefore, whether there are some deviations needs to be evaluated and improved through further clinical studies.

\section{Abbreviations}

GC: Gastric cancer

ROC: Receiver operating characteristic

EGE: Electronic gastroscopy examination

PG: Pepsinogen

ELISA: Enzyme-linked immunosorbent assay

Hp: Helicobacter pylori

AUC: Area under curve

PGR: Ratio of PG I to II

HL: Hosmer-Lelneshow

NOC: N-nitroso compounds. 


\section{Data Availability}

The data used to support the findings of this study are included within the article.

\section{Conflicts of Interest}

The authors declare no conflicts of interest.

\section{Authors' Contributions}

Wei Tao and Li Yang contributed to the design of the study; Yu-Feng Guo and Peng Li collected, analyzed, and interpreted the data. Wei Tao, Hai-Xia Wang, and Li Yang drafted and revised the manuscript. All the authors read and approved the final manuscript.

\section{Acknowledgments}

The authors would like to thank all patients who participated in this study and everyone who contributed to this article.

\section{References}

[1] F. Bray, J. Ferlay, I. Soerjomataram, R. L. Siegel, L. A. Torre, and A. Jemal, "Global cancer statistics 2018: GLOBOCAN estimates of incidence and mortality worldwide for 36 cancers in 185 countries," CA: a Cancer Journal for Clinicians, vol. 68, no. 6, pp. 394-424, 2018.

[2] L. Yang, R. Zheng, N. Wang et al., "Incidence and mortality of stomach cancer in China, 2014," Chinese Journal of Cancer Research, vol. 30, no. 3, pp. 291-298, 2018.

[3] L. Zakko, L. Lutzke, and K. K. Wang, "Screening and preventive strategies in esophagogastric cancer," Surgical Oncology Clinics of North America, vol. 26, no. 2, pp. 163-178, 2017.

[4] L. Flores-Luna, M. M. Bravo, E. Kasamatsu et al., "Risk factors for gastric precancerous and cancers lesions in Latin American counties with difference gastric cancer risk," Cancer Epidemiology, vol. 64, article 101630, 2020.

[5] F. Ceu, C. Susana, K. Andreas, and M. J. Carlos, "Pathogenesis of gastric cancer," Helicobacter, vol. 20, Supplement 1, 2015.

[6] L. H. Eusebi, A. Telese, G. Marasco, F. Bazzoli, and R. M. Zagari, "Gastric cancer prevention strategies: a global perspective," Journal of Gastroenterology and Hepatology, vol. 35, no. 9, pp. 1495-1502, 2020.

[7] K. Sun, R. Zheng, and S. Zhang, "Report of cancer incidence and mortality in different areas of China, 2015," China Cancer, vol. 28, no. 1, 2019.

[8] National Health Commission Of The People's Republic Of China, "Chinese guidelines for diagnosis and treatment of gastric cancer 2018 (English version)," Chinese Journal of Cancer Research, vol. 31, no. 5, pp. 707-737, 2019.

[9] Y. Wang and Q. Wang, "Key points and difficulties in prevention and treatment of chronic disease-interpretation of guidelines for prevention and treatment of chronic disease in China (2017-2025)," Academic Journal of Second Military Medical University, vol. 38, no. 7, pp. 828-831, 2017.

[10] J. Yin, X. Wu, S. Li, C. Li, and Z. Guo, "Impact of environmental factors on gastric cancer: a review of the scientific evidence, human prevention and adaptation," Journal of Environmental Sciences, vol. 89, no. 3, pp. 65-79, 2020.
[11] T. Slavin, S. L. Neuhausen, C. Rybak et al., "Genetic gastric cancer susceptibility in the international clinical cancer genomics community research network," Cancer Genetics, vol. 216-217, pp. 111-119, 2017.

[12] R. W. Kneller, W. D. Guo, A. W. Hsing et al., "Risk factors for stomach cancer in sixty-five Chinese counties," Cancer Epidemiology, Biomarkers \& Prevention, vol. 1, no. 2, 1992.

[13] E. Denova-Gutiérrez, R. U. Hernández-Ramírez, and L. LópezCarrillo, "Dietary patterns and gastric cancer risk in Mexico," Nutrition and Cancer, vol. 66, no. 3, pp. 369-376, 2014.

[14] A. P. Thrift and H. B. El-Serag, "Burden of gastric cancer," Clinical Gastroenterology and Hepatology, vol. 18, no. 3, pp. 534-542, 2020.

[15] T. Wei, Establish Gastric Cancer Scoring Models of High-Risk Population and Study the Opportunistic Screening Method of Gastric Cancer, Ningxia Medical University, 2012.

[16] X. Yang, J. Ge, H. Cai, and Y. Ge, "Study on correlation of dietary habits and risk of gastric cancer in Hui population," Modern Preventive Medicine, vol. 39, no. 11, pp. 2674-2676, 2012.

[17] Y. Fujino, A. Tamakoshi, Y. Ohno, T. Mizoue, N. Tokui, and T. Yoshimura, "Prospective study of educational background and stomach cancer in Japan," Preventive Medicine, vol. 35, no. 2, pp. 121-127, 2002.

[18] H. Gao, N. Li, and Q. Zhang, "Diagnostic value of serum PGI, PGII and G-17 in gastric cancer and atrophic gastritis," Oncology Progress, vol. 15, no. 6, pp. 654-656, 2017.

[19] E.-J. Cho, H.-K. Kim, T.-D. Jeong et al., "Method evaluation of pepsinogen I/II assay based on chemiluminescent immunoassays and comparison with other test methods," Clinica Chimica Acta, vol. 452, pp. 149-154, 2016.

[20] G. A. Colditz, K. A. Atwood, K. Emmons et al., "Harvard report on cancer prevention volume 4: Harvard Cancer Risk Index. Risk Index Working Group, Harvard Center for Cancer Prevention," Cancer Causes and Control, vol. 11, no. 6, pp. $477-488,2000$.

[21] K. Otani, T. Teshima, Y. Ito et al., "Risk factors for vertebral compression fractures in preoperative chemoradiotherapy with gemcitabine for pancreatic cancer," Radiotherapy and Oncology, vol. 118, no. 3, pp. 424-429, 2016.

[22] S. Babiker, O. Nasir, S. H. Alotaibi, A. Marzogi, M. Bogari, and T. Alghamdi, "Prospective breast cancer risk factors prediction in Saudi women," Saudi Journal of Biological Sciences, vol. 27, no. 6, pp. 1624-1631, 2020.

[23] N. Alsheridah and S. Akhtar, "Diet, obesity and colorectal carcinoma risk: results from a national cancer registry-based middle-eastern study," BMC Cancer, vol. 18, no. 1, article 1227, 2018.

[24] Z. Wenbin, F. Yang, and L. Zhaoshen, "How to improve the diagnosis rate of early gastric cancer in China," Journal of Zhejiang University (Medical Sciences), vol. 44, no. 1, pp. 9-14, 2015. 